\title{
Stability and Energy Aware Multipath Routing for Mobile Ad Hoc Networks
}

\author{
P.Srinivasan \\ Dept. of Computer Applications \\ Mahendra Inst. of Technology \\ Namakkal
}

\author{
P. Kamalakkannan,Ph.D \\ Dept. of Computer Science \\ Government Arts College \\ Salem
}

\author{
S.P.Shantharajah,Ph.D \\ Dept. of Computer Applications \\ Sona College of Technology \\ Salem.
}

\begin{abstract}
Multipath routing protocols for Mobile Ad hoc NETwork (MANET) addresses the problem of load balancing, scalability, security and life time of networks. In this paper, we propose a new multipath protocol, Stability and Energy Aware Multipath Ad hoc On-demand Distance Vector (SEAM-AODV) protocol which is an enhancement of Ad hoc On-demand Distance Vector (AODV) protocol. It designs a bi-objective optimization formulation to compute the reliability factor based on the stability and residual energy of nodes, through cross layer approach. The reliability of the multiple paths is increased through node disjointness. The path with the highest reliability factor value is selected as the primary path for data transmission. It also employs effective route maintenance mechanism to reduce the frequency of route recovery. This protocol is compared with other similar routing protocol: AOMDV. We use ns-2 for simulation. Our simulation results show that, the proposed protocol reduces the packet loss by up to $25-35 \%$ and routing overhead by about 32- $40 \%$. It also accomplishes $10-13 \%$ higher packet delivery ratio.
\end{abstract}

\section{Keywords}

MANET, Multipath routing, route stability, bi-objective optimization.

\section{INTRODUCTION}

Mobile ad hoc networks (MANET) are groups of wireless mobile devices, which can communicate with each other without any infrastructure support. It is a self-configured and self-maintained network with no central authority. Every node in MANET acts as both a host and a router. Dynamic topology, limited bandwidth, battery, CPU resources and multi-hop communication are the characteristics that put special challenges in routing protocol design.

Several routing protocols have been proposed for MANETs. Based on the route discovery principle, we can classify them into either proactive or reactive. Proactive routing protocols update routes for every pair of nodes at regular intervals irrespective of their requirement. Higher bandwidth consumption and extra overhead due to regular route updates are the disadvantage of these protocols. The reactive or ondemand routing protocols, determine route only when there is a need to transmit a data packet, using a broadcasting queryreply (RREQ-RREP) procedure. It is found that the routes discovered by these protocols are not stable, resulting in frequent link breakages.
The ad hoc routing protocol can be classified as single path routing or multipath routing, based on the number of routes computed between source and destinations. Single path routing selects a shortest path between the source and destination for data communication. It has to invoke a new route discovery whenever the single path fails. Each route discovery is associated with latency, packet loss and overhead. Hence, it is desirable to keep the route discovery frequency low.

Multi-path routing protocols [1-6] establish multiple disjoint paths from a source to a destination. The main objectives of multipath routing protocols are to provide reliable communication and to ensure load balancing. The frequency of route discovery is very low in multipath routing protocols. It goes for route discovery when the entire path fails. Therefore, the frequency of route discovery is very low in multipath routing protocols.

The stability based routing protocols are designed to choose stable route passing through stable links. These protocols improve route lifetimes and packet delivery ratio compare to the shortest path routing protocols. The energy-aware routing protocols are designed considering factors like residual energy, total transmission power or both. These protocols avoid over using of certain nodes and reduce total energy consumptions. But, there exist a very few protocols in the literature that consider both stability and energy metric during route discovery and maintenance.

In this paper, our objective is to develop a new on-demand multipath routing protocol called stability and energy aware multipath ad hoc on-demand distance vector (SEAM-AODV) protocol. SEAM-AODV is based on the most widely used ondemand routing protocol ad hoc on-demand distance vector (AODV) protocol. SEAM-AODV protocol extends the AODV protocol to discover multiple paths in every route discovery. It ensures that multiple paths discovered are loopfree and node disjoints paths. The route discovery and maintenance is based on the stability and energy metrics, which considerably reduce the frequency of route discoveries.

This paper is organized as follows. In section 2, we present related work on stability and energy aware routing protocols. Section 3 describes the proposed scheme SEAM in detail. Section 4 presents the Stability and Energy Aware Multipath routing protocol (SEAM-AODV). Finally, simulation results and conclusions are summarized in section 5 and 6 , respectively. 


\section{RELATED WORK}

This section introduces some related contributions on multipath, link stability based protocol, energy aware protocols and a few papers on joint stability-energy metric based protocols.

AODV [15] a reactive routing protocol establish a route to a destination only on demand and destination sequence numbers are applied to find the latest route to the destination. The advantage of AODV is reduced control overhead compare to proactive routing protocols. However, it discovers only a single path for data communication.

Multipath routing protocols provide multiple routes that can be used to support dynamic .topology changes in MANET. These protocols show better performance in terms of packet delivery ratio, routing overhead and end-to-end delay.

Split Multi-path routing (SMR) [3] is an extension to Dynamic source routing. Instead of dropping duplicate RREQ, it forwards the duplicate RREQ packet through a different incoming link that have less hop count value than the earlier received RREQ. It does not take traffic load into account which results in invoking congestion. In SMR-LB [4], each intermediate node records how many primary paths are attempted. Each intermediate node decides which path should be constructed by using the source node ID and the primary path on it already. So it can avoid a large amount of traffic on a certain node.

Ad hoc On-demand Multi-path Distance vector routing (AOMDV) [5] extends AODV to provide multiple path. Unlike AODV, every RREP is being considered by the source node and thus multiple paths discovered in one route discovery. The intermediate node maintains multiple path entries in their respective routing table. Every node maintains an advertised hop count for the destination. Advertised hop count defined as the "Maximum hop count for all the paths". Route advertisements of the destination are sent using this hop count. An alternate path to the destination is accepted by a node if the hop count is less than the advertised hop count for the destination. However, it does not consider stability or energy metrics of nodes during route discovery.

AODV_Multipath is an extension of AODV protocol designed to find multiple node-disjoint paths. In [6] the authors measure route quality in terms of SINR, which gives reliable link. However, the overall route stability is not considered.

Link stability is a measure of how stable the link is and how long it will last. Signal strength, pilot signals, link duration distributions, residual energy of the nodes and relative speed between nodes are the parameters used for the computation of link stability. Stability based routing protocols use link stability factor and path stability factor calculated using the above specified parameters to select stable path for data transmission. The connection failure probability based stability calculation approach monitors the links lifetime in the past, to predict its behavior in the future without considering parameters depend on mobility models. They quest to maximize the stability with constrained path length for improving the route discovery in MANET [7-10].

The lifetime of a network is one of the important factors to be considered in designing a MANET routing protocol. Maximizing the network lifetime by minimizing the power consumption for the data transfer is the main aim of Energy aware routing protocols [11-13]. Optimizations carried out in these protocols are classified in the following schemes. (i)
Minimize the total energy consumed along the route (ii) Avoid using node with minimum residual energy. They achieve energy efficiency by optimizing the node radio range based on the geographical distance. They notably reduce the energy wastage ensuing from retransmission due to bit error rate, frame error rate and link failures due to energy depletion.

In [14], the authors propose Link-Stability and Energy Aware Routing protocol (LEAR). It considers joint metric of link stability and energy drain rate into route discovery. It balances the traffic load on the nodes and considerably decreases the control overhead. However, LAER does not able to discriminate between links of the same age.

\section{STABILITY AND ENERGY AWARE (SEA) MODEL}

The problem of selecting a reliable path in mobile ad-hoc network is formulated as a bi-objective optimization problem. This section describes the metric definitions and multiobjective problem formulation.

\subsection{Stability Metric}

SEAM model considers signal strengths and node mobility for computing the probability of link failures. It utilizes the extended device driver interface to get the signal strength information from the device. Then it computes link stability (LS) using signal strength values received from the MAC layer.

Any link $e=(i, j) \quad \mathrm{E}$ has an associated link stability $L S(e)$ and it is given by

$$
\mathrm{LS}_{\mathrm{i}, \mathrm{j}}=\frac{\mathrm{u} 2-\mathrm{DSS}_{\mathrm{i}, \mathrm{j}}}{\mathrm{u} 2-\mathrm{u} 1}
$$

where $\mathrm{DSS}_{\mathrm{i}, \mathrm{j}}$ is the differentiated signal strength to decide whether the signals are getting stronger or weaker. It is computed as follows.

$$
\operatorname{DSS}_{i, j}=\operatorname{SScur}_{i, j}-\text { SSnew }_{i, j}
$$

Formally, a path between two nodes $\mathrm{s}$ and $\mathrm{d}$ is a set of all feasible path between them and can be represented as $P(s, d)$ $=\{P 0, P 1, \ldots, P n\}$ where each $P_{i}$ is a feasible path between $s$ and d.

We define the stability of the path $\mathrm{P}$, by the product of link stability of its edges [11] as follows

$$
\operatorname{Stability}(\mathrm{P})=\prod_{\mathrm{e} \in \mathrm{P}} \operatorname{LS}(\mathrm{e})
$$

The path with higher path stability value contains more stable links and choosing it will considerably reduce the probability of link failure.

\subsection{Energy Metric}

The energy metric of the path is given by

$$
\operatorname{EM}(\mathrm{P})=\prod_{\mathrm{i}=1}^{\mathrm{n}}\left(\frac{\mathrm{R}_{\mathrm{i}}(\mathrm{t})}{\mathrm{F}_{\mathrm{i}}}\right)
$$

where $R_{i}(t)$ is remaining battery capacity and $F_{i}$ is full battery capacity of intermediate node $i$, at time $t$. The goal of this metric is to maximize EM 


\subsection{Problem Formulation}

The problem can be stated as - "To find multiple paths for data communication based on route stability and residual energy metrics.

The above bi-objective optimization problem can be transformed into a single objective problem, by providing importance factor (i.e. w1 and w2) for each criterion of the objective. We combine the objectives into a single objective function to calculate the Reliability Factor (RF) of the path P, can be mathematically stated as

$$
\mathrm{RF}(\mathrm{P})=\mathrm{w}_{1} . \text { Stability }(\mathrm{P})+\mathrm{w}_{2} \cdot \operatorname{EM}(\mathrm{P})
$$

where the parameters $w 1$ and $w 2$ are chosen based on the network dynamics and application requirements. In this study, in order to give equal importance to both stability and energy metrics, we assign 0.5 to both $w 1$ and $w 2$, such that $w 1+w 2=1$ condition is satisfied.

\section{STABILITY AND ENERGY AWARE MULTIPATH ROUTING PROTOCOL}

The proposed routing protocol SEAM-AODV uses route stability and energy metrics of intermediate nodes for route selection. It uses the stability and energy model discussed in section 3 for computing the stability and energy metrics.

SEAM-AODV computes a maximum of three node-disjoint routes from the source to the destination and uses the route with maximum stability metric as the primary path and the other as the secondary paths. It computes node-disjoint path to increase the likelihood that though the primary path fails, the source can find the alternate path remain valid. Selecting routes with higher stability as the primary route increases the reliability of the discovered route. It comes with "makebefore-break" route maintenance mechanism. This mechanism quickly adapt to the link breakage likely to occur due to the mobility and energy drain, by switching to one of the alternate paths. . A new route discovery is initiated only when all the paths fail.

\subsection{Route Discovery}

The most popular reactive routing protocol AODV is modified to include an additional mechanism to discover multiple node-disjoint paths for a source and destination pair. The RREQ of SEAM-AODV is an extension of a RREQ packet of AODV routing protocol. Two new fields Accumulated Path Stability (APS) and Accumulated Energy Metric (AEM) are added to the RREQ Packet. It initializes the values to the added fields as follows: APS, AEM with 1.

It limits the number of RREQ forwarded by an intermediate using the advertised hop count value. It selectively forwards the duplicate RREQ to reduce the path diminution. It forwards RREQ packets with a hop count less than or equal to the advertised hop count and coming through a distinct first hop neighbor of the source or through the same first hop neighbors of the source with a better reliability value are forwarded by the intermediate node. This advertised hop count value is stored in the reverse routing table (RRT).

The intermediate node on receiving the RREQ, checks whether the signal strength and residual energy is greater than the threshold value. If the above conditions are satisfied, then node i make a reverse route entry in the Routing Table (RT). Then it calculates LS. If the signal strength is above SThr1, then it assigns 1 to LS. It implies that nodes are close and, link is sufficiently stable. Otherwise, it calculates LS using equations 1 and 2. Energy metric is calculated using equation 4. It stores the stability and advertised hop count information in the Route Forward Table (RFT), such that the updated values contain the route stability and energy metric of the explored route up to the current node using equations 3 and 4 .

Source starts a timer of duration RREP_WAIT_TIME. In case it does not receive any RREP message before the timer expires, it reinitiates the route discovery process repeatedly for utmost MAX_RREQ_TIMEOUT until it receives the RREP message as in AODV. It increases the back off period exponentially each time when the route discovery process fails.

\subsection{Route selection at the destination node}

Destination node will receive RREQ packets from different possible routes. It checks the signal strength and computes the route stability as performed by an intermediate node. On receiving the first RREQ packet, the node $\mathrm{D}$ starts a timer $\Delta \mathrm{t} 1$ for the duration of Route Reply Latency (RRL) time $(0.3 \mathrm{sec})$. With some experimental simulations, we found $0.3 \mathrm{sec}$ was enough time to get sufficient RREQ from different paths. It computes RF value for the path explored by the RREQ. It stores all the RREQ that arrives along with its reliability factor and first node ID, in its routing table. The destination is allowed to store at most one RREQ per first hop neighbor to restrict the maximum number to be handled by the destination. After the timer $\Delta \mathrm{t} 1$ expires, the destination sorts the RREQ in descending order of their reliability factor (RF) values calculated using equation 6 .

Then it selects at most three node-disjoint paths from the sorted RREQ packets depending on the availability of the paths. The selected paths are the most reliable node-disjoint paths. The destination unicast RREP to the source through the selected paths. The intermediate nodes on receiving the RREP packet make the forward and reverse entry in the RT. The timeout period is set to automatically purge the entries in case there are no data or RouteM packets within the expiry of timeout period. The reverse entries are used to return RouteM packets back to the source node and are active as long as the forward entry is active.

The source node on receiving the RREP packet makes an RT entry. It selects the path with the highest reliability factor as the primary path and the remaining as the secondary paths. It marks the corresponding values (primary or secondary) in the associated flag field on the routing table. It starts the data transmission on the primary path.

\subsection{Route Maintenance}

After the initiation of data transmission the maintenance of the primary path and secondary path is important. If an intermediate node detects any route failures then it informs the source by sending the RERR packet. All intermediate nodes receiving the RERR packet delete the corresponding entry from their routing table and forward it towards the source. The source node on receiving the RERR packet removes the corresponding entry from its routing table and checks whether it is through the primary route or secondary route. If it is through the primary route then it will switch to the most reliable secondary route immediately. If it is through the secondary route then it will go for the next secondary route if available. 


\subsection{An Example}

\section{Route Request Forward Table (RFT) at E}

\begin{tabular}{|l|l|l|l|}
\hline Seq. No., Src, Dst & FID & APS & AEM \\
\hline 100, S, D & A & .56 & .81 \\
\hline 100, S, D & B & .9 & .86 \\
\hline
\end{tabular}

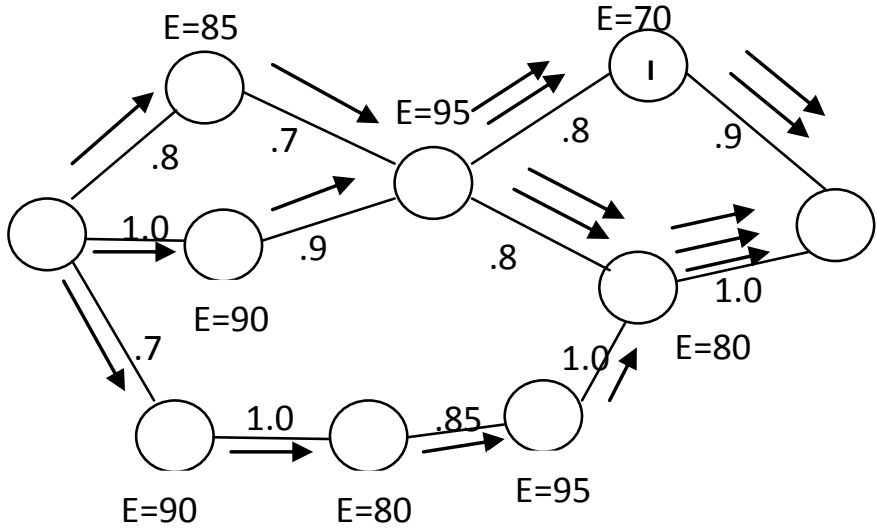

Fig 1: Route Establishment in SEAM-AODV

Figure 1 shows the node-disjoint multipath route discovery process of SEAM-AODV for a network of 10 nodes. In the figure, the labels represent the current link stability and residual energy of the intermediate nodes. Node $S$ wants to communicate with Node D; it does not have a route to the destination in its route cache. RSEA-AODV protocol initializes 1 to the fields APS and AEM in the RREQ packet. The Node $S$ broadcasts RREQ packet to discover route to the destination D.

The intermediate nodes on receiving the RREQ, process it as discussed in section 4.2. If the stability and energy metrics are satisfied, then it updates the APS and AEM fields based on the strength of the packet it received and the residual energy of the node.

The number of arrows represents the number of route request packets forwarded over that particular link. It makes an entry of first node Id, APS and AEM information on the route forward table (RFT) for implementing the RREQ forwarding policy discussed in section 4.2. The RFT entries made in node $\mathrm{E}$ is shown in the figure $\mathrm{x}$. The destination node $\mathrm{D}$ receives five RREQ packets within the timer $\Delta$ tlexpires. It stores the relevant information from the RREQ packet into the destination's route list table (RLT). It selects node disjoint paths from that list. The shaded entries S-B-E-H-D and S-CF-G-H-D are the node disjoint paths identified by the SEAMAODV. Destination D sends RREP to source $S$ through the nodes I and $\mathrm{H}$.

The RREP packet contains the path information and stability values in it. On receiving the RREP packets, node $S$ determines the path with higher reliability S-B-E-H-D as the primary path and the other path S-C-F-G-H-D as the secondary path and starts data transmission through the primary path. Source sends RouteM packet at regular intervals during data transmission to keep the secondary path active

\begin{tabular}{|c|l|l|l|l|}
\hline $\begin{array}{c}\text { Seq. No., Src, } \\
\text { Dst }\end{array}$ & FID & \multicolumn{1}{|c|}{ Path } & APS & AEM \\
\hline 100, S, D & B & S-B-E-H-D & .72 & .65 \\
\hline 100, S, D & B & S-B-E-I-D & .65 & .60 \\
\hline 100, S, D & C & S-C-F-G-H-D & .60 & .56 \\
\hline 100, S, D & A & S-A-E-H-D & .48 & .65 \\
\hline 100, S, D & A & S-A-E-I-D & .43 & .57 \\
\hline \hline
\end{tabular}

Route List Table (RLT) at D

and to measure the stability values of both the primary and secondary paths.

\section{SIMULATION AND PERFORMANCE EVALUATION}

In this section, we inspect the performance of RSEA-AODV routing protocol and compare it with the similar protocols PERRA and AODV.

\subsection{Simulation Parameters}

We simulate it in NS2 2.31 [16]. We run the simulation for 10 times and the results show $95 \%$ confidence interval on all observed metrics. The simulation parameters are listed in table 1 .

Table 1. Simulation parameters

\begin{tabular}{lll}
\hline Parameter name & Value & \\
\hline Topology & $1000 \times 500 \mathrm{~m}$ & \\
Number of nodes & 50 & \\
$\begin{array}{l}\text { Mobility model } \\
\text { point }\end{array}$ & Random $\quad$ Way \\
Transmission range (m) & & \\
Simulation time (s) & 250 & \\
Number of flows & 600 & \\
Traffic type & 10 & \\
Traffic Rate (packets/sec) & CBR & \\
Packet size (B) & 10 & \\
Node speed & 512 & \\
P1,P2 & $0,5,10,15,20$ & \\
0.9 & $0.1,0.3,0.5, \quad 0.7$, \\
SThr1 & & \\
SThr2 & $1.5 \times \mathrm{R} \times \mathrm{Thr}$ & \\
\hline
\end{tabular}


From figures 2 and 3, it is observed that the packet delivery ratio (PDR) of SEAM-AODV improves considerably in comparison with AOMDV. The PDR of SEAM-AODV is more than $96 \%$ in all mobility cases. But, the PDR of AOMDV reduces up to $82 \%$.

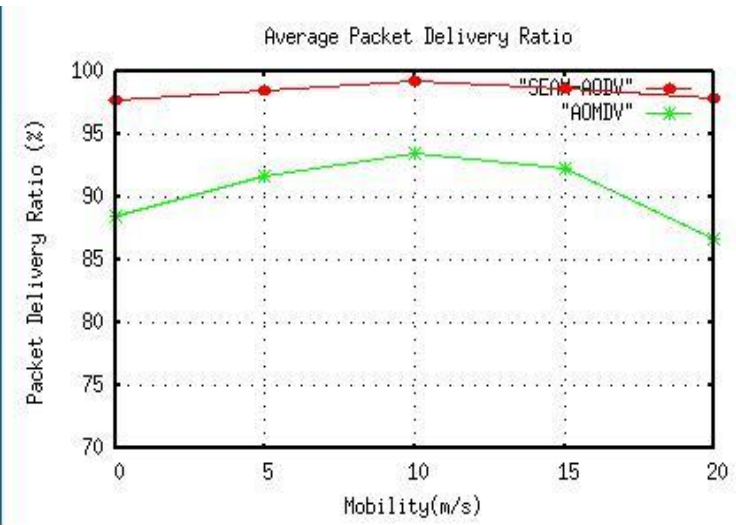

Fig 2: Packet Delivery Ratio vs. Mobility

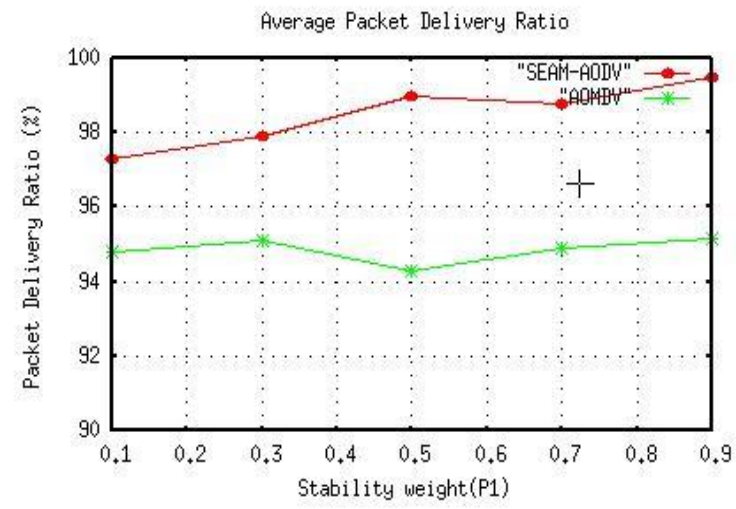

Fig 3: Packet Delivery Ratio vs. Stability

This is because of route selection and, route maintenance strategy carried on by the SEAM-AODV. Its RREQ forwarding policy considers the stability and residual energy of the intermediate nodes. In case of AOMDV, it chooses a path with the shortest route as the primary route. It may contain low energy nodes which lead to disconnections of sessions.

Similarly, SEAM-AODV shows better performance for different stability weights $(\mathrm{P} 1)$ with the maximum PDR of $99.25 \%$ at stability weight of 0.9 . It is observed that the PDR increases as the stability increases. This is because stability is given more importance than the energy metrics as the stability increases. But, AOMDV maintains an average packet delivery ratio of $95 \%$. It does not show any discrepancy due to stability changes as it does not consider stability of the nodes during route selection.

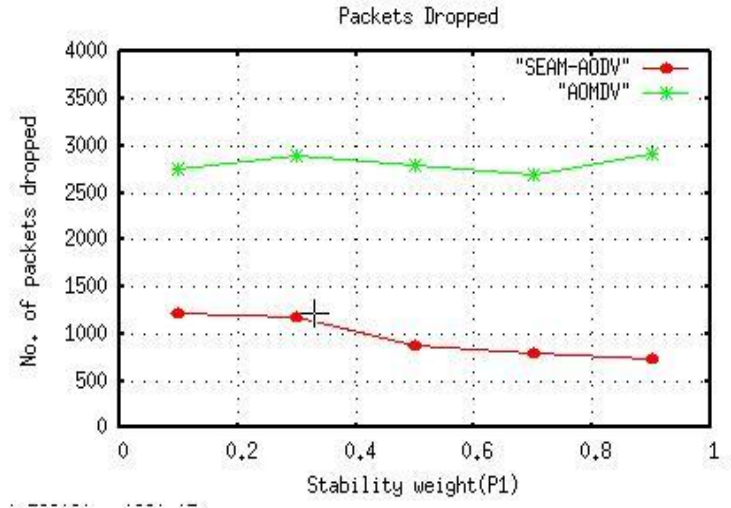

Fig 4: Packet dropped vs. Stability

From figures 4 and 5 , it is observed that the number of packets dropped during data communication in SEAMAODV is less compare to AOMDV in all mobility and stability cases. This is achived by means of route maintanence mechanism adapted by the SEAM-AODV. In most of the cases, the primary route is switched before it breaks due to node movement or energy drain. From figure 4, it is also observed that as the stability weight increases there is a decrease in the number of packet dropped. This is due to selection of stable paths which reduces the probability of link breakages. The amount of packet loss in AOMDV is two to three times more than that of the SEAM-AODV.

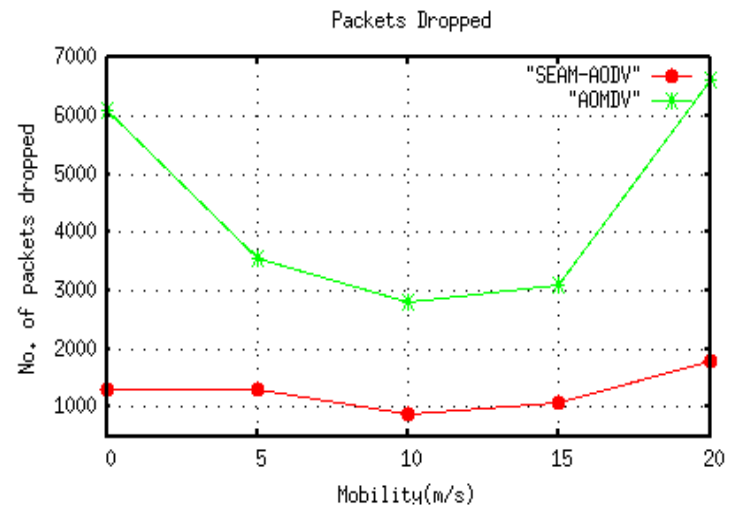

Fig 5: Packet dropped vs. Mobility

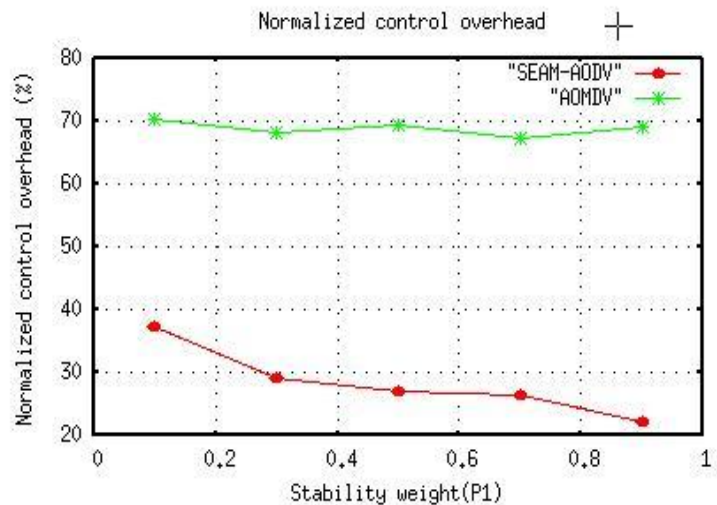

Fig 6: Control Overhead vs. Stability 


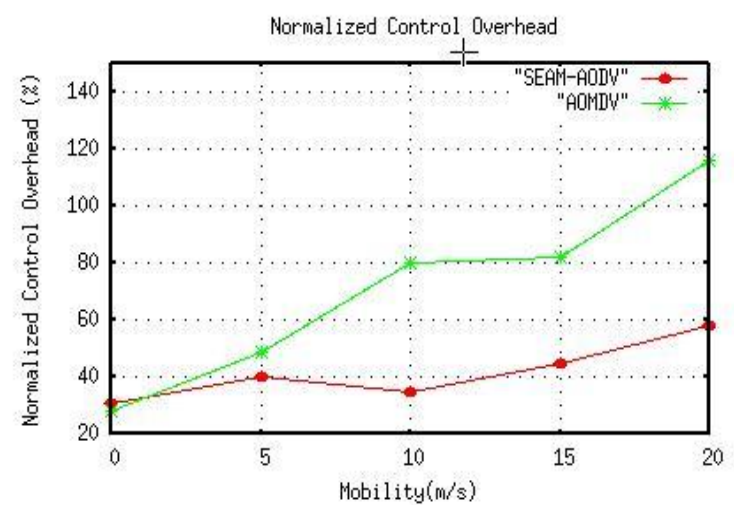

Fig 7: Control Overhead vs. Mobility

From figures 6 and 7, it is observed that the control overhead of SEAM-AODV remains lower in all mobility and stability conditions due to its selection of stable routes and maintenance of alternate path. It is noted that there is a gradual increase in the control overhead as the mobility increases in AOMDV, due to increase in route recovery. In case of SEAM-AODV, there is a gradual decrease in the control overhead as the stability weight increases. This is because more importance is given to stability rather than the energy metrics, resulting in reduced route breakage and reconstruction. . Though the route maintenance overhead is not present in AOMDV, the control overhead of it is higher. This is due to its route selection procedure. It selects routes that are unstable which fail after a short period of their discovery leading to frequent route discoveries by the source node.

Table 2. Simulation results

\begin{tabular}{|c|c|c|c|c|c|}
\cline { 3 - 6 } \multicolumn{2}{c|}{} & \multicolumn{2}{c|}{ AOMDV } & \multicolumn{2}{c|}{ SEAM-AODV } \\
\cline { 3 - 6 } \multicolumn{1}{c|}{} & $\begin{array}{c}\text { Throughput } \\
\text { (kbps) }\end{array}$ & $\begin{array}{c}\text { Delay } \\
(\mathbf{m s})\end{array}$ & $\begin{array}{c}\text { Throughput } \\
\text { (kbps) }\end{array}$ & $\begin{array}{c}\text { Delay } \\
(\mathbf{m s})\end{array}$ \\
\hline \multirow{4}{*}{ Mobility } & $\mathbf{0}$ & 291 & 98.1 & 334 & 12.6 \\
\cline { 2 - 6 } & $\mathbf{5}$ & 340 & 105.3 & 356 & 13.5 \\
\cline { 2 - 6 } & $\mathbf{1 0}$ & 362 & 96.8 & 404 & 11.9 \\
\cline { 2 - 6 } & $\mathbf{1 5}$ & 332 & 118.2 & 376 & 20.3 \\
\cline { 2 - 6 } & $\mathbf{2 0}$ & 316 & 144.6 & 336 & 24.4 \\
\hline \multirow{5}{*}{ Stability } & $\mathbf{0 . 1}$ & 331 & 79.1 & 384 & 14.97 \\
\cline { 2 - 6 } & $\mathbf{0 . 3}$ & 325 & 78.02 & 395 & 13.12 \\
\cline { 2 - 6 } & $\mathbf{0 . 5}$ & 363 & 82.6 & 410 & 12.47 \\
\cline { 2 - 6 } & $\mathbf{0 . 7}$ & 354 & 94.4 & 399 & 11.96 \\
\cline { 2 - 6 } & $\mathbf{0 . 9}$ & 375 & 88.5 & 435 & 12.07 \\
\hline
\end{tabular}

Table 2 gives the simulation results. The average network throughput obtained by SEAM-AODV is $11 \%$ and $13 \%$ higher than AOMDV in mobility and stability cases respectively. This is achieved by reducing the amount of packet drop and unnecessary control overhead due to path breakage. It is observed that in all mobility and stability conditions, SEAM-AODV maintains an average end-to-end delay below $21 \mathrm{~ms}$. But in AOMDV, the delay is not bounded and reaches up to $150 \mathrm{~ms}$ and $1 \mathrm{~s}$ for mobility and stability cases, respectively. Though finding a reliable route considering the stability and energy metrics increases the delay on the one hand. On the other hand, route selection and route maintenance procedure of SEAM-AODV increases the lifetime of the routes and the bottle-neck nodes, thus significantly reducing the need for packet retransmissions.

\section{CONCLUSIONS}

In this paper, we proposed a new Stability and Energy Aware Multipath routing protocol. It establishes route based on the joint metric of link stability and residual energy. The use of stability model and energy metric considerably reduces the number of route recoveries during data transmissions. Simulation results show SEAM-AODV perform better in terms of packet delivery ratio, control overhead and end - to end delay, especially in high mobility scenarios. The discovery of route failures and switching to available alternate route before actual route break saves route recovery time and reduces the packet loss during data transmission.

\section{REFERENCES}

[1] Tarique, M., Tepe, E., Sasan Adibi, and Shervin Erfani. 2009. Survey of multipath routing protocols for Mobile Ad-hoc networks. Journal of Network and Computer Applications, 32:1125-1143.

[2] Mona Gupta and Neeraj Kumar. 2013. Node-Disjoint On-demand Multipath Routing with route Utilization in Ad-Hoc Networks. International Journal of Computer Applications.

[3] Lee,S.J. and Gerla, M. 2001 .Split Multipath Routing with Maximally Disjoint paths in Ad Hoc Networks. In proceedings of IEEE ICC.

[4] Sharma, D.K., Sanjay Kumar and Chiranjev Kumar 2010. Enhancement of Split Multipath Routing protocol in MANET. International Journal on Computer Science and Engineering, 2: 679-685.

[5] Marina, M.K., and Das. S. 2001. On-Demand Multipath distance vector routing in adhoc Networks. In proceding of the international conference on network protocols.

[6] Park, and Chung, I. 2011. Multipath AODV routing protocol in Mobile Ad hoc with SINR based route selection. Wireless Comm. Systems, 4:682 - 686 .

[7] Abid, M. and Belghith. A. 2011. Stability routing with constrained path length for improved routability in dynamic MANETs. Personal and Ubiquitous Computing , 15:799-810.

[8] Hui, Z. and Ning, D.Y. 2007. A novel path stability computation model for wireless ad hoc networks. IEEE Signal Process. Letter, 14:928-931.

[9] Ho, A. H. and Hua, K. A. 2009. Handling high mobility in next-generation wireless ad hoc networks. Int. J. Commun. Systems, 23:1078-1092.

[10] Nityananda Sarma and Sukumar Nandi. 2010. Multipath QoS Routing with Route Stability for Mobile Ad Hoc Networks. IETE Technical Review, 27:380-397.

[11] Tan and Bose, S. K. 2012. Power and mobility aware routing in wireless ad hoc network. Commun, IET, 6:1425-1437.

[12] Ashish Kumar and Kamal bansal. (2012). Energy efficient Routing Protocol Avoiding Route Breaks based on DSR," International Journal of Computer Applications, 44:39 -44.

[13] Park and Lee, S. 2008. A routing protocol for Extent Network Lifetime through the Residual Battery and Link Stability in MANET. In Proc. ACC '08, 199-204. 
[14] Rango, F. De., Fazio, P., and Guerriero, F. 2012. Link Stability and Energy Aware Routing Protocol in Distributed Wireless Networks. IEEE Trans. on Parallel and Distributed systems, 23: 713-726.
[15] Perkins, C. E., Royer, E. M. and Das, S. R. 2003. Ad hoc on-demand distance vector routing, IETF RFC 3561.

[16] The NS simulator and the documentation [Online]. Available from: http://www.isi.edu/. 